\title{
Les Marabouts africains à Paris
}

\author{
Liliane Kuczynski
}

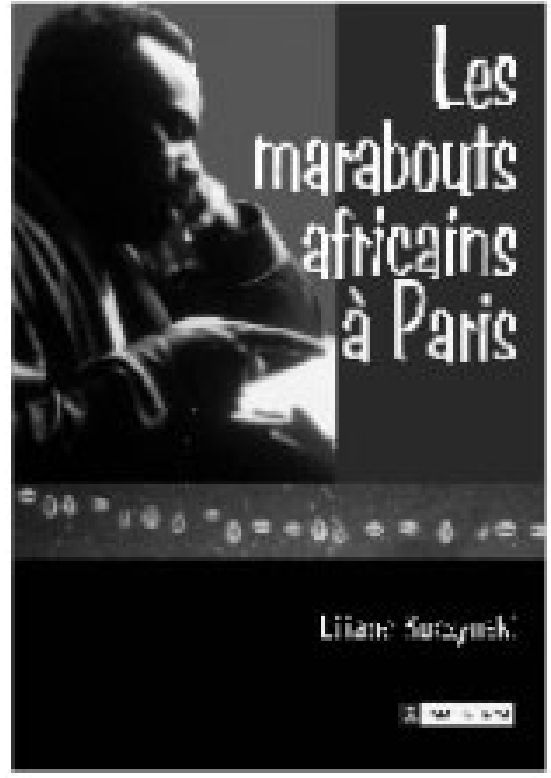

Cet ouvrage résulte d'une longue enquête conduite à Paris et dans sa banlieue auprès des marabouts originaires d'Afrique de l'Ouest et de leurs clients. Après avoir analysé le rôle protéiforme des marabouts, les raisons de leur venue en France, la diversité de leur cheminement individuel, l'auteur ètudie leur implantation dans la région parisienne, puis expose leurs pratiques liées à la divination et à la résolution de al'infortune et du malheuro tout comme les méthodes de constitution de leur clientèle diversifiée.

A la frontière de plusieurs cultures et plusieurs mondes, ceux du Nord et du Sud, de la religion, de la magie, de la voyance, les marabouts africains jouent un rôle charnière entre communautés immigrées et populations autochtones et témoignent de l'existence d'un islam ordinaire.

Collection CNRS Anthropologie 2003 - $17 \times 24-420$ p - illustré - br. $27 €$

Pour trouver et commander nos ouvrages :

LA LIBRAIRIE de CNRS ÉDIIONS, 151 bis, rue Saint-Jacques - 75005 PARIS Tél. : 0153100505 - Télécopie : 0153100507 - Mél : librairie@cnrseditions.fr

Site Internet : www.cnrseditions.fr

Frais de port par ouvrage : France : $5 €$ - Elranger : $5,5 €$

Pour plus de renseignements, n'hésitez pas à contacter

le Service clientèle de CNRS ÉITIONS, 15, rue Malebranche - 75005 Paris

Tél : 01531027 07/09 - Télécopie : 0153102727

Mél : cnrseditions@enrseditions.fr

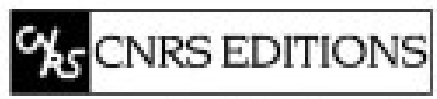

un editeur au service de frédition scientiflque 


\section{Revue française de sociologie}

publiée avec le concours du

CENTRE NATIONAL DE LA RECHERCHE SCIENTIFIQUE

et de l'INSTITUT DE RECHERCHE SUR LES SOCIÉTÉS CONTEMPORAINES

59-61, rue Pouchet 75849 Paris Cedex 17 - Tél. : 0140251187 ou 88

Alcoolisation et alcoolisme Pierre MERLE dans un centre de tri postal Bertrand LE BEAU

Conflits, styles d'interactions conjugales et milieu social

Éric WIDMER Jean KELLERHALS René LEVY

De l'ouvrier à l'opérateur: Sylvie MONCHATRE chronique d'une conversion

Du recours au paradigme épidémiologique

Patrick PERETTI-WATEL pour l'étude des conduites à risque

École publique, école privée Chloé TAVAN Trajectoires et réussite scolaires

\section{LES LIVRES}

Abonnements/Subscriptions (2004) :

L'ordre et le paiement sont à adresser directement à :

Please send order and payment to:

Éditions OPHRYS BP 8705003 GAP cedex France 0492538572

France :

Particuliers : $80 €$ (4 numéros trimestriels)

Institutions : $90 €$ (4 numéros trimestriels)

Institutions : $110 €$ (4 numéros trimestriels + supplément en anglais)

Étudiants : $\quad 60 €$ (4 numéros trimestriels)

Étranger/Abroad :

$110 €$ (4 numéros + supplément en anglais/

four quarterly issues + the English selection)

Vente au numéro/Single issue :

Le numéro trimestriel/for each quarterly issue : $23 €$

La sélection anglaise/for the English selection : $30 €$ 


\title{
Revue de l'histoire des religions
}

http://rhr.revues.org

Publication trimestrielle fondée en 1880

\author{
DIRECTION \\ C. AMIEL, J. SCHEID \\ RÉDACTION \\ C. AMIEL
}

CONSEIL DE RÉDACTION

G. AUDISIO, L. BANSAT-BOUDON, N. BELAYCHE

P. BOURETZ, P. ELLINGER, S. GEORGOUDI

J. LE BRUN, F. LESTRINGANT, P. LORY

M. OLENDER, H. WISMANN

CORRESPONDANTS À L'ÉTRANGER

M. BETTINI (Sienne), P. BORGEAUD (Genève)

B. FAURE (Stanford), M. HARA (Tokyo)

M. IDEL (Jérusalem), A. SANDERSON (Oxford)

H. D. KITTSTEINER (Francfort-sur-l'Oder)

\section{$1 / 2004$}

Dumézil encore, toujours. Which Triad ? A Critique and Development of Dumézil's Tripartite Structure (Emily LYLE) - Tullus Hostilius et le thème indoeuropéen des trois péchés du guerrier (Dominique BRIQUEL). - Recherches bibliques. Pourquoi le Horeb après le Sinaï ? (Jean KOENIG). - Les Juges d'Israël : une invention du Deutéronomiste ? (Isabelle de CASTELBAJAC)

\section{$2 / 2004$}

Le dieu Thot et la parole (Youri VOLOKHINE) — Les dieux du cycle de la régénération dans quelques figures celtiques (Adolfo ZAVARONI) - Préserver l'action au sein de la clôture : le compromis des Ursulines de Toulouse (16041616) (Laurence LUX-STERRITT) - De l'étal à l'église : l' "Union Professionnelle Catholique de la Boucherie » (1930-1980) (Sylvain LETEUX) Inquisition, juifs et nouveaux-chrétiens dans les capitaineries du Nord de l'État du Brésil aux XVII ${ }^{\text {ème }}$ et XVIII ${ }^{\text {ème }}$ siècles (Bruno FEITLER)

$$
* * *
$$

CORRESPONDANCE : Revue de l'histoire des religions, Collège de France, 11 place Marcelin Berthelot, 75231 PARIS Cedex 05, E-mail : rhr@ehess.fr

VENTE ET ABONNEMENTS : Presses Universitaires de France, 6, avenue Reille, 75685 Paris Cedex 14, E-mail : revues@puf.com

Abonnement 2004 (4 fascicules), France : $64 €$ Etranger : $77 €$, Prix au numéro : $21 €$ 


\section{STUDI STORICI}

rivista trimestrale dell'Istituto Gramsci

\section{4}

Gli espazis del tardoantico

Elio Lo Cascio, Premessa

Glen W. Botversock, Riflessioni sulla periodizzazione dopo «Esplosione di tardoanticos di Andrea Giardina

Lellia Cracoo Ruggini, Come e perché è sesplosom il tardoantico?

Arnaldo Marcone, La tarda antichità o della difficoltà delle periodizzazioni

Aldo Schiavone, Piccolo esperimento mentale in tre sequenze

Andrea Giardina, Tardoantico: appunti sul dibattito attuale

Opinioni e dibattiti

Anna Maria Rao, Il giacobinismo italiano nell'opera di Carlo Zaghi

Silvio Pons, L'aaffare Gramsci-Togliattis a Mosca (1938-1941)

Giorgio Vercellin, Tolleranza islamica e tolleranza occidentale: confronti e cambiamenti

\section{Ricerche}

Adolfo La Racca, Liberi e schiavi nella prima guerra servile di Sicilia

Carla Ferrante-Antonello Mattone, Le comunità rurali nella Sardegna medievale (secoli XI-XV)

Renata Segre, La potestà giurisdizionale sugli ebrei nell'Italia di antico regime: diritto e magistrature

Beatrice Alfonzetti, Eugenio eroe perfettissimo. Dal canto dei Quirini alla rinascita tragica

Note critiche

Mario Gaglione, Allusioni gioachimite nella basilica angioina di Santa Chiara a Napoli?

Giovanni Miccoli, La vita e i tempi di Pietro Perna

\section{Libri ricevuti}

abbonamento annua $\in 50,50$ (singoli). $€ 55,90$ (biblioteche $e$ istituzioni), $€ 66,50$ (estero), ocp 77228005 - Carocci editore spa, via Sardegna 50, 00187 Roma, tel. 0642014260 , fax 0642747931. $\theta$-mail riviste Ecarocci.it indirizzo della redazione: Fondazione Istituto Gramsci Onlus, Via Portuense 95c, 00153 Roma, tel. 06 5806646, fax 065897167 , e-mail studistorici@i fondazionegramsci.org 


\section{Ammales du Midi revue de la France méridionale}

№247, Juillet-Septembre 2004

CURSENTE (Benoît), Les abbadies (abbayes laïques).

Dîme et société dans les pays de l'Adour $\left(\mathrm{XI}^{\mathrm{e}}-\mathrm{XVI}^{\mathrm{e}}\right.$ siècles).

FRITZ (Jeanne-Marie), La vicomté de Marsan : naissance et fossilisation

d'une grande seigneurie gasconne au Moyen Age.

VIAUD (Jean-François), La maladie dans les mémoires et les diaires de l'Ancien Régime :

exemples dans le Bordelais au début du XVIII ${ }^{\mathrm{e}}$ siècle.

BERLAN (Hélène), La mobilité étudiante au XVIII ${ }^{\mathrm{e}}$ siècle :

l'exemple de la Faculté de médecine de Montpellier

DÉBAX (Hélène) et MACÉ (Laurent), Deux sceaux inédits des Trencavel

\section{No 248 - Octobre-Décembre 2004}

BAÏSSE (Élisabeth), Les princes de Conti en Languedoc au milieu du XVII ${ }^{\mathrm{e}}$ siècle

WHITLOCK (Stéphanie), Le commerce cultivé : les allées de Tourny et le Jardin royal à Bordeaux au XVIII ${ }^{\mathrm{e}}$ siècle

CARLUER (Jean-Yves), Un grand éditeur protestant du XIX ${ }^{\mathrm{e}}$ siècle, la Société des livres religieux de Toulouse.

LORCIN (Marie-Thérèse), La micro-toponymie du Vivarais dans les estimes de 1464 : une première approche.

RENTET (Thierry), Louis de Fourmillon, premier capitaine du château d'If ( $d$ 'après les lettres conservées au musée Condé de Chantilly). 


\section{E T U D E S \\ R U R A L E S \\ $n^{*} 167-168$ \\ Juillet-decembre 2003}

\section{OBJETS EN CRISE, OBJETS RECOMPOSÉS}

\section{Gérard Chouquer}

Introduction

François Favory

1. a part de l'indigtne et du vernaculaire dans les iextes des arpenteurs romains

\section{Maria Conceição Lopes}

Réflexions sur le modele de la cite antique : l'exemple de Pax halia (Beja, Portugal)

Gerard Chouquer

Lespace des sociebés antiques, entre poojet et expérienoe Claire Marchand

Des centuriacions plus belles que jamais ?

Propxitice d'un modele dynamique d'organisation des formes

Sandrine Robert

Coenment les formes du passed se transmettent elles?

Celdrie I avigne

De nouveaux objets d'hisloire agraire porar eth finir avec le bocage et l'openfield

Magali Watteaux

1e plan radio-quadrille des termoirs esn planifies

Joeille Burnouf

La Nature des médievistes

Melanie Foucault

Dynamique d'un corridor a fluviaire * sur la commune Les Maillys (COe-d $\mathrm{Cr}$ )

Caroline Pinoteau

Changer la carte, c'est changer l'objet

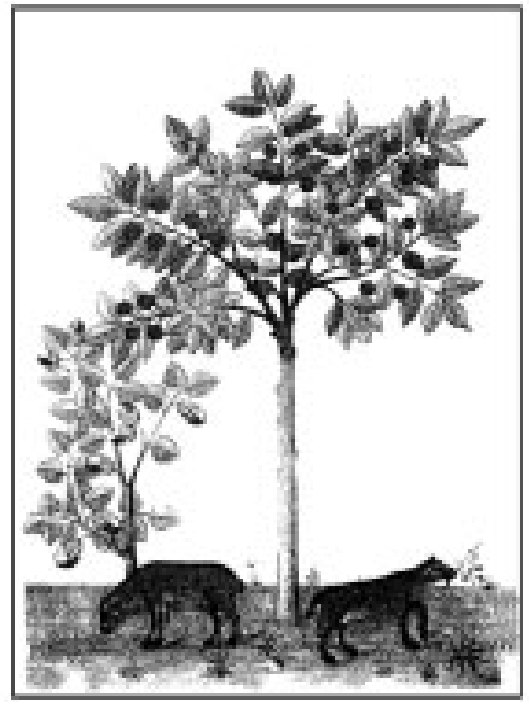

Caroline Pinoteau et Franersea Di Pietre

Association de formes et de dynamiques dans le bassin-versant de P'Aubricre (l-dre-et-Loire) Claire Delhon, Fanny Moutarde, Marzareta Tengherg et Stéphanie Thiebault

Perceptions ef reprtsentations de l'espace à traver les analyses archeobotaniques

Glarsaine

A propos

Magali Watteas

A propos de la = naissance du village au Moyen Ảge * : la fin d'un paradzme?

\section{Antowr do thene}

Campdes rendus

\section{$\phi$}

\section{Rbelinction}

Latoratoine of antropetepie seciake

52. ne du Candinal Leneine

79005 Pinis

ItL. : $0144271743 / 44$

$\mathrm{fax}: 0144271766$

tadk ruralo Gehas if

\section{Abenarnents}

FHESS - Service iboencuretis

5.7, me Mavelis Berthelot

92762 Anecy codes

th. : 0155595253

ax $=0155595250$

abo serviseses wandos fr

huriation:

Frace : $64.50 \%$ noteranger i 7450 \& $\pi x$ Paricaliery !

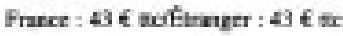

\section{Veate au aumero}

CID - 131, bid Saist-Michal

75605 Pari

새. 10143544715

fax : 0143540073

cidenok-pins fir

sumero simpte $15,30 \mathrm{C}$

armere double $32 \epsilon$

numtro triple 396 


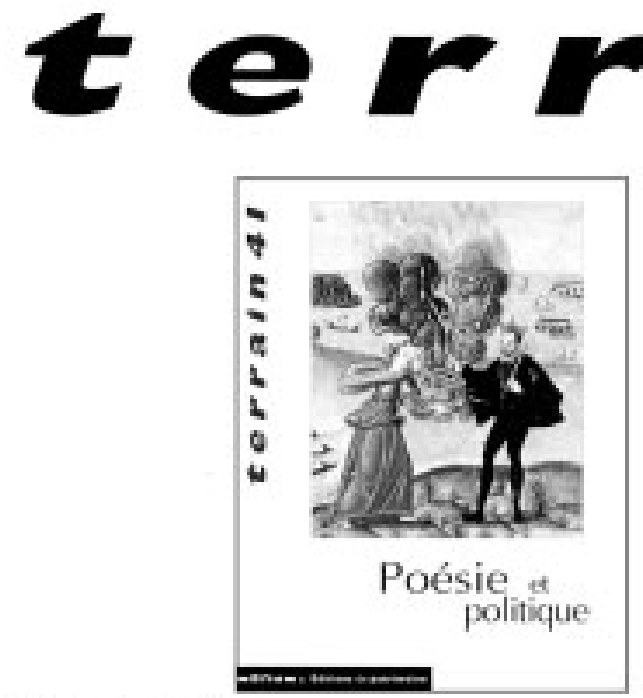

Mission a I'Ethnokgrie

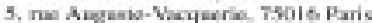

TeI. 133 i0)1 535753 19 - Fex $=33$ i001 $5357 \times 3.33$

Mel. : caristinc. larebiselsediure.fr

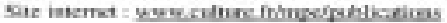

DIRECTRUCF

DE IA PUBLICATTOS

Ohate Wirlicle

READCTKKCE EN CHEF

Christime Lavglay

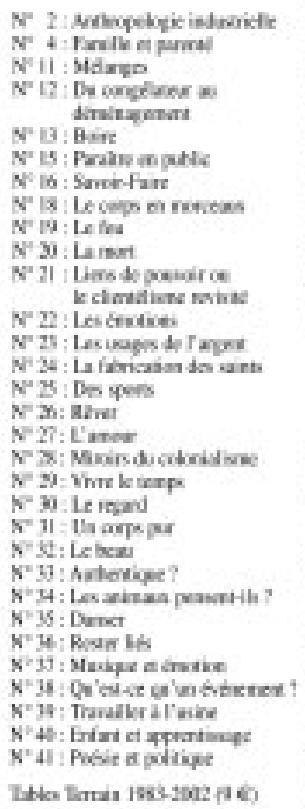

Terrain parait semestricllement. Son ambition est de mieax faire connaine kes étades ethnologiques bu comaine frangais et europen notamment celles reali. stes avec le concours de la mission du Patrimoine ethnologique.

Le dososier themalique est asowarti de la rubrique e Repleres - qui accueille des anticles hors thème et d'anIres iraitant de la valorisaticen de la recherehe, airesi gae des = Infos = sar l'achualiet de l'elhnolagie de l'Fursore.

Berus arestricle.

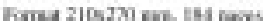

Vera wa cordon pis is $24 \epsilon$

it $4 \mathrm{E}$ de fras de peos

indorma an:

CID, 131, st 5aira-Mkgt.

Tyes Pain

Ta 137 int 435443 is

Fas: 9310014554 ks 75

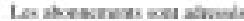

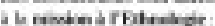

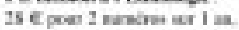

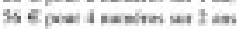

Pumber a raneted:

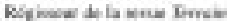

manum teditiens da parrimeine

(2)

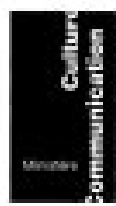

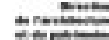

sommaire

TEMAIN 41 i SETEMBAE 2003

Terrown, vingt ans après

\section{POÉSIE ET POLITIQUE}

\% Solitaine et solidaire w

Gitretien avec Edouand Gissant

Philippe Artières.

Poésie officielle, poetsle partisane pendant les guerres de Religion

- Tariana Debtage Baranova

Les prèteres de la langue

Potsic, nation et politique en Serbic

Iman Colovic

* Une politique qui wole sur les ailes de la poésie *

Pratiques politico-podaiques au sein de la Lieue du

Nond

Martina Avanca

Poésie idéologique et espace de fiberté en Roumanie Duxia Dragomir

a Poésie et civisme n en Trégor

Emmonuele Calac

Révolutionnaire ou nationaliste?

La poesie ocotane apres 1968

Dilppe Marte

Qui sait danser sur cette chanson,

nous lui donnerons la cadence

Musique, poessie et politique chez les Touareys

Nada Betalmar

\section{REPERES}

L'esprit ensorcele

Les racines cogritives de la sorcelerie

Fabrice Cxemen

Urbanisme et cuartier

Ce que nous apprend Paris Five gaurche

Alain Bourdin

Le territoire urbain

Divisions et spatislsations

Resemarie Huhn et Alain Marr

MESUMES - ABSTRACTS

INFOS 


\section{Techniques GQ culture}

$$
\begin{gathered}
\text { Numéro } 41 \text { - Janvier-juin } 2003 \\
\text { Briques : le cru et le cuit }
\end{gathered}
$$
A. Guillerme
Présentation
C.-A. de Chazelles
C. Bodolec données archéologiques

V. Nègre chinoises (XIVe-XXe siècles)
A. Montigny
et S. Sanz
Maisons en terre archéologique à Mahasthangarh
(Bangladesh)

\section{VARIA}
P. Reigniez Les baguettes à récolter du Caucase
V.Lanougùre-Brineau Le garde-manger de Mota Lava (Vanuatu) : un meuble bien particulier
C. Hincker Du beau à l'identité. Représentations touarègues de l'expression esthétique

Témoignages croisés sur les constructions antiques en terre crue : textes latins et

La terre crue dans la construction publique des deux dernières dynasties

La "Théorie-pratique " du pisé. Mise en discours et en images d'une technique et sa réception dans le Sud-Ouest de la France aux XVIIIe et XIXe siècles

Chroniques \& commentaires

F.-R. Picon Ce qu'esclave veut dire. A propos du livre d'Alain Testart : L'esclave, la dette et le pouvoir. Études de sociologie comparative

J. Ivanoff La mort de l'ethnologie ? Réflexions à partir l'ouvrage Ethnologie. Concepts et aires culturelles

\begin{tabular}{|c|c|c|}
\hline & \multicolumn{2}{|c|}{ Abonnement $41-42$} \\
\hline $\begin{array}{l}\text { France } \\
\text { Etranger }\end{array}$ & $\begin{array}{c}\text { Particulier } \\
27 \text { euros } \\
30 \text { euros }\end{array}$ & $\begin{array}{r}\text { Institution } \\
30 \text { euros } \\
35 \text { euros }\end{array}$ \\
\hline
\end{tabular}

\section{CONDITIONS DE VENTE}

Vente au numéro - prix du numéro 15 euros (frais d'envoi en sus)

S'adresser au

CID, 131 boulevard Saint-Michel, 75005 PARIS

Tél. 01435447 15, Fax 0143548073

Libeller les chèques à l'ordre du CID 


\section{L'Homme et la Société}

Revue internationale de recherches et de synthèses en sciences sociales

N 143-144

\section{Compétences pour résister}

Les armes du faible sont-elles de faibles armes?

(Marc BESSIN, Laurence ROULLEAU-BERGER)

Tassadit YACINE, Domination, résistance et représentation : Lecture'de fables kabyles

Geneviève PRUVOST, Les "inommables » de la préfecture de Police

Lilian MATHIEU, Quand «la peur devient une existence »: sur la place de la violence dans le monde de la prostitution

Isabelle BERRY-CHIKHAOUI, Agnès DEBOULET, L'enjeu de la question des compétences des citadins. Illustration sur le Monde arabe

Roch HuRTUBise et Michèle VATz LAAROUSSI, Réseaux, stratégies et compétences : Pour une analyse des dynamiques sociales à

$l^{\prime} œ u v r e$ chez les jeunes de la rue

Michèle LECLERC-OLIVE, Territorialités de migrants

Claudia BARRIL, L'art de l'autre : nouvelles pratiques de contact et initiatives de publicité des Mapuches du Chili

Denis MERKLEN, Le quartier et la barricade : Le local comme lieu de repli et base du rapport au politique dans la révolte populaire en Argentine

Smaïn LAACHER, Faire de la politique sans expérience : des étrangers au parlement européen

Guillaume MARCHE, Le mouvement gai et lesbien américain face au sida

Comptes rendus. Revue des revues. Abstracts.

Publié avec le concours du Centre national du Livre et du Centre national de la Recherche scientifique

AвONNEMENTS ET VENTES AU NUMÉRO : Éditions L'Harmattan

5-7 rue de l'École-Polytechnique 75005 PARIS

France : $47,26 €$ - Étranger par avion : 53,36€

Un n simple : $13,72 €$, double : $18,29 €+3,20 €$ de port

Un abonnement annuel couvre 3 numéros dont 1 double 


\section{économie appliquée}

Tome LVI - $N^{\circ} 4$ - décembre 2003

Olivier BRETTE

Fabrice LEQUEUX, Jérôme GALLO

Christian BESSY

Jan GROOT,

Loek GROOT

Meixing DAI,

Moïse SIDIROPOULOS

Gabriel TAHAR
L'accumulation des connaissances technologiques dans la pensée de Thorstein Veblen : ressources pour l'analyse évolutionniste contemporaine

Effets d'interdépendance économique et convergence stratégique dans l'industrie multimédia

Distributed Cognition and the Theory of the firm :

Enriching Nelson and Winter's Evolutionary Analysis of Organisational Learning and Memory

The Competitive Balance of French Football, 1945-2002

Règle du taux d'intérêt optimale, prix des actions et taux d'inflation anticipé : une étude de la stabilité macroéconomique

\section{NOTES ET COMMENTAIRES}

Frotière de compétence et fonction de gain : un réexamen des rendements de l'éducation

ISSN : 0013-0494 - Prix public : $29 €$ Les Presses de I'ISMÉA - BP 22 - 75622 Paris cedex 13 


\section{Éditions de I'E.H.E.S.S.}

\section{CAHIERS DES ANNALES}

Ouvrages disponibles en librairie

Diffusion : Armand Colin - distribution : Livredis

S. ALBERRO, Les Espagnols dans le Mexique colonial (1992).

G. BÉAUR, L'immobilier et la Révolution. Marché de la pierre et mutations urbaines, 17701810 (1994).

R. GUESNERIE, F. HARTOG (sous la direction de), Des Sciences et des Techniques : un débat (1998).

P. A. KUHN, Les origines de l'État chinois moderne, traduit et présenté par P.-É. Will (1999).

P. JEANNIN, Change, crédit et circulation monétaire à Augsbourg au XVIe siècle (2001).

Diffusion-distribution : C.I.D.

M. AGULHON, Le cercle dans la France bourgeoise 1810-1848 (1977).

P. CHAUNU, Histoire quantitative, histoire sérielle (1978).

W. G. L. RANDLES, De la terre plate au globe terrestre (1980).

S. L. KAPLAN, Le complot de famine : histoire d'une rumeur au XVIII' siècle (1982).

R. HALÉVI, Les loges maçonniques dans la France d'Ancien Régime (1984).

R. E. GIESEY, Cérémonial et puissance souveraine, France, $X^{\mathrm{e}}-\mathbf{X V I I}$ siècles (1987).

B. MÜLLER, Bibliographie des travaux de Lucien Febvre (1990). 


\section{Abonnement 2004 / Subscription 2004}

$\square$ Je souhaite souscrire un abonnement d'un an (6 numéros) à / I want to subscribe for one year (6 issues) to :

$\square$ Je souhaite souscrire un abonnement de deux ans (12 numéros) à / I want to subscribe for two years (12 issues) to :

\section{Annales. Histoire, Sciences sociales}

\begin{tabular}{lcc} 
& \multicolumn{2}{c}{ FRANCE / DOM-TOM } \\
Tarifs & $\mathbf{1}$ an $\left(\mathbf{6} \mathbf{~}^{\circ \mathbf{s}}\right)$ & $\mathbf{2}$ ans $\left(\mathbf{1 2} \mathbf{~ n}^{\mathrm{os}}\right)$ \\
Particuliers / Individuals & $\square 78,50 €$ & $\square 135 €$ \\
Institutions / Institutions & $\square 95,00 €$ & $\square 163 €$ \\
Étudiants / Students* & $\square 61,00 €$ & \\
$*$ Sur présentation de la carte / Student card required &
\end{tabular}

Prénom / First name

Nom / Name

Adresse / Address

Code postal / Postal code

Ville / Town

Pays / Country

Choisissez votre mode de paiement / Please check how you wish to pay

$\square$ Directement, par chèque, Eurochèque / Directly, by cheque, Eurocheque

$\square$ Par carte de crédit / By credit card: $\square$ CB $\quad \square$ Visa $\square$ Master Card / Eurocard

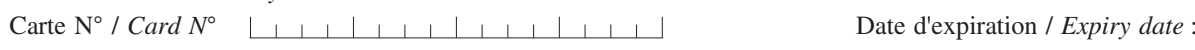

$\square$ Envoyez-moi un reçu / Please, send me a receipt Signature :

Adressez votre commande et votre paiement à l'ordre de / Send your order and payment to :

COLIN-ABONNEMENTS - F 75704 PARIS CEDEX 13

Date de validité de l'offre : 31/12/2004

— Les abonnements sont mis en service dans un délai maximum de quatre semaines après réception de la commande et du règlement / Subscriptions begin 4 weeks following receipt of payment.

— Les abonnements partent du premier numéro de l'année / Subscriptions begin with the first issue of calendar year.

— Les réclamations pour les numéros non reçus doivent parvenir dans un délai maximum de six mois / Claims may be submitted to the publisher for missing issues for a đ̇ period of six months after publication of each individual issue.

— Numéros séparés de l'année et volumes antérieurs / Back issues and volumes (jusqu'à épuisement du stock) :

COLIN-ABONNEMENTS - F 75704 PARIS CEDEX 13

Tél. : Numéro Indigo : 0820065095 (France) - 33140648902 (Étranger) - Courriel : abonnement@editions-sedes.com 


\section{$\mathrm{N}^{0} 5-6$ \\ à paraître automne 2004}

\section{Asie centrale}

Pierre Chuvin

Denise Aigle

Bakhtyar Babajanov

Stéphane A. Dudoignon

Dru C. Gladney

Frantz Grenet

Masami Hamada

Fürgen Paul

Olivier Roy

Maria Szuppe

Éric Trombert

Etienne de la Vaissière

\section{Mondes musulmans}

